\title{
Frontal fibrosing alopecia and lichen planus pigmentosus: diagnosis and therapeutic challenge*
}

\author{
Fabiane Andrade Mulinari-Brenner ${ }^{1}$ \\ Murilo Calvo Peretti ${ }^{1}$
}

\author{
Marina Riedi Guilherme ${ }^{2}$ \\ Betina Werner ${ }^{3}$
}

DOI: http:/ /dx.doi.org/10.1590/abd1806-4841.20175833

\begin{abstract}
Frontal fibrosing alopecia is a variant of lichen planopilaris with marginal progressive hair loss on the scalp, eyebrows and axillae. We report a case of frontal fibrosing alopecia and lichen planus pigmentosus in a postmenopausal woman, that started with alopecia on the eyebrows and then on the frontoparietal region, with periocular and cervical hyperpigmentation of difficult management. The condition was controlled with systemic corticosteroid therapy and finasteride. Lichen planus pigmentosus is an uncommon variant of lichen planus frequently associated with frontal fibrosing alopecia in darker phototipes. It should be considered in patients affected by scarring alopecia with a pattern of lichen planopilaris and areas of skin hyperpigmentation revealing perifollicular hyperpigmentation refractory to multiple treatments. This case illustrates diagnostic and therapeutic challenge in face of scarring alopecia and perifollicular hyperpigmentation.
\end{abstract}

Keywords: Alopecia; Hyperpigmentation; Lichen planus

\section{INTRODUCTION}

One of the clinical types of lichen planus (LP), first described in India by Bhutani and Bedi, is lichen planus pigmentosus (LPP). ${ }^{1}$ It is a rare cutaneous pigmentary disorder of unknown etiology, with female predominance, between the third and fourth decades of life. ${ }^{2}$ Currently, many publications show the association of LPP and frontal fibrosing alopecia (FFA). ${ }^{1,3-5}$

FFA, a lichen planopilaris variant, with identical histological features, is characterized by a frontal or frontotemporal band of alopecia, associated to the loss of eyebrows and body hair. Lichen planopilaris is more frequent in Caucasian adult women, with an unknown etiology and variable pattern of hair loss, with foci of partial scarring alopecia. ${ }^{6}$

Scarring alopecia results in an irreversible damage to the hair follicles, with progressive and permanent hair loss. We present the case of a patient with FFA and LPP, with control of the latter after treatment of the alopecia.

\section{CASE REPORT}

A 59-year-old dark-skinned postmenopausal woman was undergoing follow-up in the Hair and Cosmetic outpatient clinic due to the complaint of alopecia in the eyebrows over the past two months, not responding to topical $5 \%$ minoxidil, and itchy periocular hyperpigmentation refractory to superficial chemical peel.

She also complained of marginal alopecia on the scalp and axillae. On examination, bilateral frontoparietal plaques of alopecia, and eyebrow (Figure 1) and axillary hair rarefaction were observed. Histopathology of the biopsy taken from the plaque revealed a pattern of scarring alopecia consistent with FFA (Figure 2). The patient was treated with three injections of triamcinolone and started chloroquine $250 \mathrm{mg}$ / day, with partial hair regrowth on the frontal region after four years and six months of follow-up. The pull test was positive and dermoscopy of the plaque revealed a scarring pattern, hair rarefaction, isolated hairs on the frontal hairline, hyperkeratosis, and blue-grey perifollicular hyperpigmentation (Figure 3).

Study submitted on 23.03.2015

Approved by the Advisory Board and accepted for publication on 24.04.2016

* Study conducted at the Department of Dermatology, Hospital de Clínicas da Universidade Federal do Paraná (HC- UFPR) - Curitiba (PR), Brazil. Financial Support: None.

Conflict of Interests: None.

Department of Dermatology, Hospital de Clínicas da Universidade Federal do Paraná (HC- UFPR) - Curitiba (PR), Brazil.

Medical School Course, Universidade Federal do Paraná (UFPR) - Curitiba (PR), Brazil.

Department of Basic Pathology, Universidade Federal do Paraná (UFPR) - Curitiba (PR), Brazil. 
Histopathology of the biopsies from the parietal alopecia plaque, eyebrow and axilla revealed a perifollicular lymphocytic inflammatory infiltrate. Subsequently, prednisone in an anti-inflammatory dose was prescribed, along with systemic tetracycline and topical betamethasone, but the patient continued with clinical hyperpigmentation on the anterior cervical region and perifollicular hyperpigmentation seen on dermoscopy in areas of the scalp, periocular and cervical regions (Figure 4). A biopsy was taken from a cervical hyperpigmented macule, and the histopathology revealed focal interface dermatitis with superficial dermal melanosis and pigmentary incontinence, consistent with LPP (Figure 5). The patient started treatment with hydroxychloroquine $400 \mathrm{mg} /$ day, with

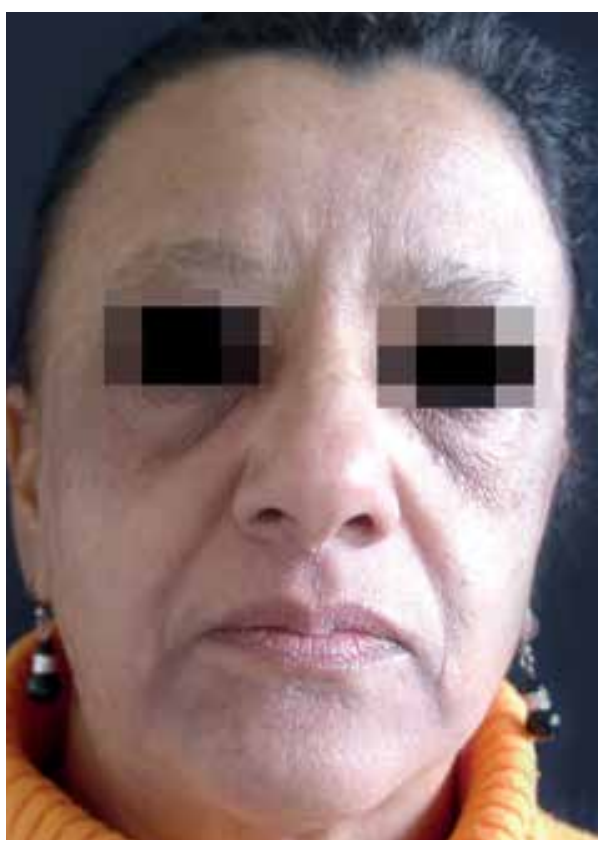

FIGURE 1: Bilateral frontoparietal alopecia and eyebrow rarefaction

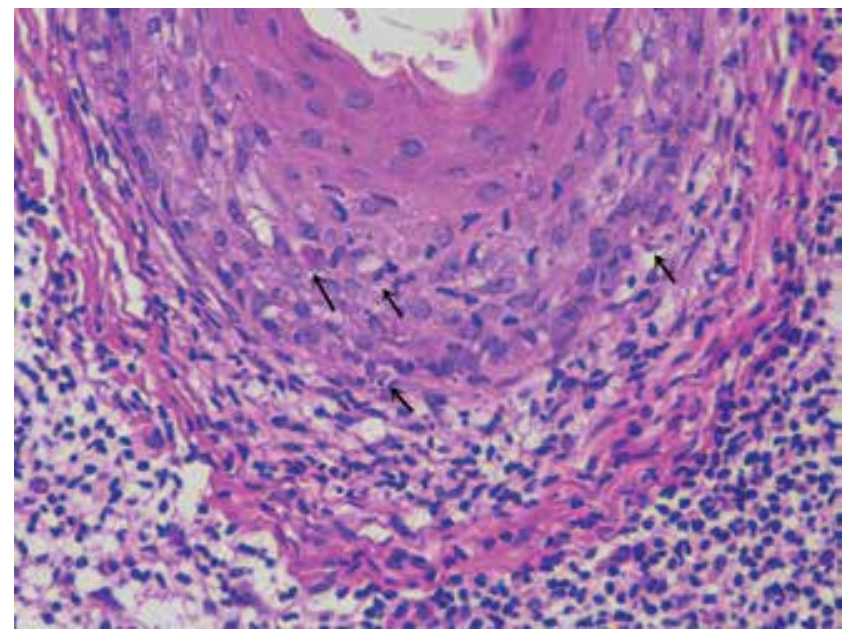

Figure 2: Biopsy of the plaque showing perifollicular fibrosis and lymphocytic infiltration in a lichenoid pattern around the follicle infundibulum and isthmus Hematoxylin \& eosin, (X400) mild improvement of the perifollicular hyperpigmentation, mild improvement in the hair growth on the eyebrows and bilateral frontotemporal area after eight months, when oral finasteride $5 \mathrm{mg}$ / day and $0.05 \%$ clobetasol were added to topical $5 \%$ minoxidil. The pigmented lesions became lighter and the patient is undergoing clinical and laboratory follow-up with partial frontal hair growth and mild eyebrow growth after 10 years of follow-up.

\section{DISCUSSION}

LPP it is a rare presentation of LP, with the clinical features of macules that range from grey to brown in photoexposed and flexural areas, mainly on the face and neck, bilateral and symmetrical, with sometimes progression to the trunk and upper limbs, usually asymptomatic, or sometimes with a burning sensation or itch, such as in our case. ${ }^{2,7}$ The etiology is unknown, and it is believed that immunological mechanisms and chemical and physical agents such as sun, mustard oil, henna hair dye and amla oil may play an important role in the pathogenesis of the disease. ${ }^{8}$

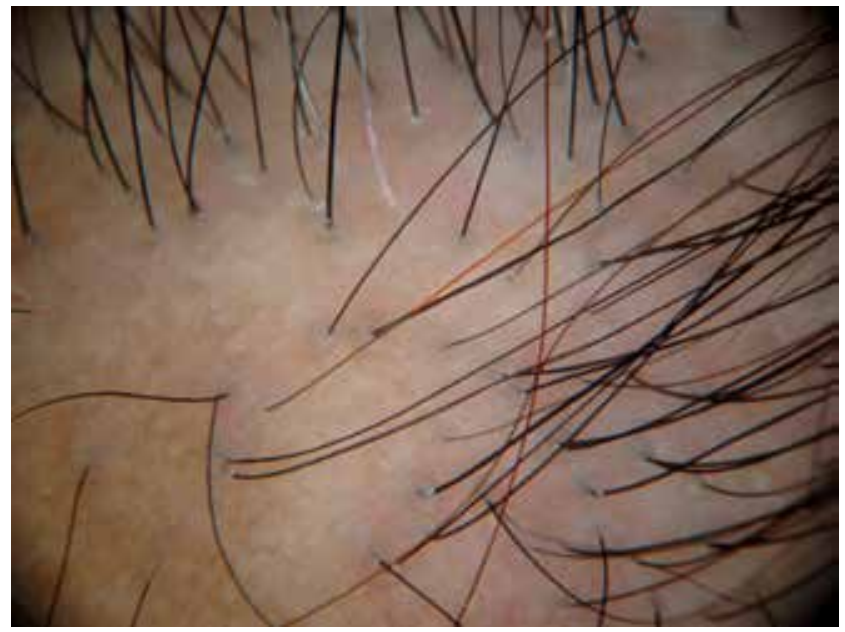

FIGURE 3: Dermoscopy of the alopecic plaque showing reduced follicular openings and perifollicular hyperkeratosis (X20)

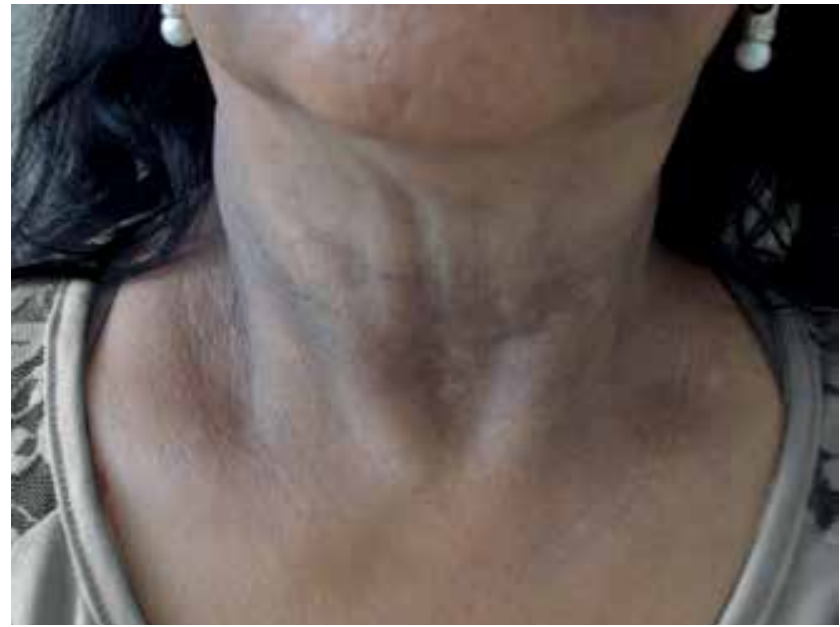

FigURE 4: Hyperpigmentation on the anterior cervical region 


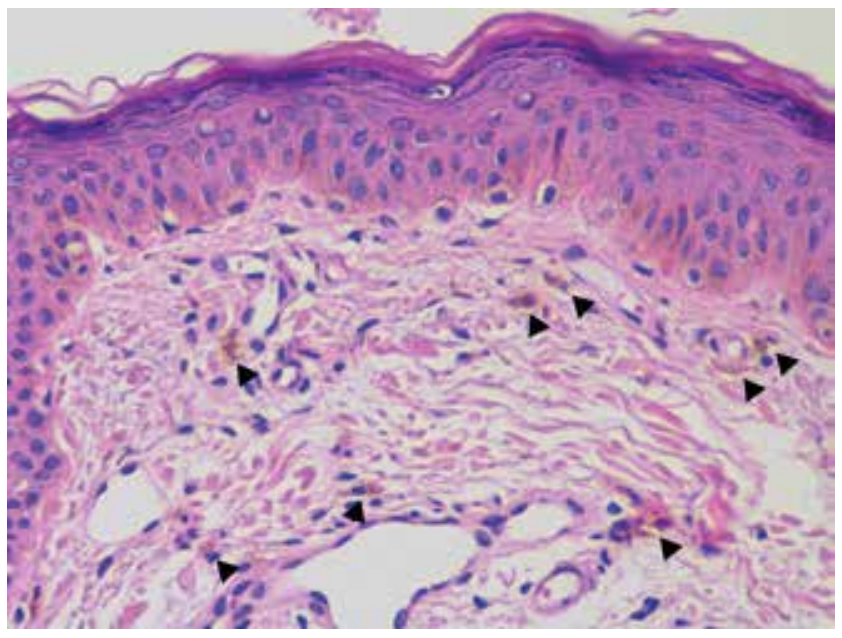

FIGURE 5: Biopsy from a cervical hyperpigmented macule with focal interface dermatitis with superficial dermal melanosis, pigmentary incontinence and presence of melanophages (arrows) Hematoxylin \& eosin, $(\mathrm{X} 400)$

The dermoscopy of LPP can reveal reduction of the follicular openings, follicular hyperkeratosis, keratotic plugs, perifollicular erythema, white dots, interfollicular vessels with single loops, arborizing vessels and pigment network. Blue-grey structures in an annular or speckled pattern can also be found and may represent basal layer degeneration, and also melanophages in the papillary dermis with signs of interface dermatitis and pigmentary incontinence. ${ }^{6,9}$ Frequent histologic findings described in the literature support the ones found in our case and include perifollicular hyperkeratosis, atrophy with hydropic or vacuolar degeneration of the basal layer of the epidermis and scarce lymphohistiocytic or lichenoid infiltrates along the dermis, with pigmentary incontinence and the presence of melanophages. ${ }^{2,3,8}$

Currently, LPP has been studied as a condition that can precede FFA. ${ }^{1,3,45}$ Initially described in Australia in 1994, FFA is a variant of lichen planopilaris, characterized by progressive scarring alopecia, with marginal and frontotemporal and eyebrow rarefaction in female patients, especially after menopause. In Brazil, the first report of groups of patients affected by the condition was from this department, with the intention to draw attention to this diagnosis. ${ }^{10}$ Scalp dermoscopy reveals loss of follicular openings, perifollicular erythema and perifollicular scales, adhered to the base of the hair shaft. The diagnosis must be confirmed with histopathology, with findings that include marked perifollicular fibrosis, lymphocytic inflammation in a lichenoid pattern around the infundibulum, isthmus and follicle bulge and reduction in the number of follicles with substitution by fibrous tracts. ${ }^{1}$

The treatment of both LPP and FFA it is still very frustrating. The management of LPP includes the use of topical medications such as steroids, immunomodulators, keratolytics, hydroquinone with or without retinoic acid, azelaic acid, kojic acid, glycolic acid, vitamin $\mathrm{A}$, aqueous solution of $10 \%$ dimethyl-sulfoxide, among others, with variable results. ${ }^{8}$ There are favorable recent reports of the use of Nd:YAG laser, intense pulsed light and dermabrasion. ${ }^{5}$

This case illustrates the diagnostic and therapeutic challenge when facing difficult alopecia management on the scalp and eyebrows and cutaneous hyperpigmentation on the periocular and cervical regions, resistant to conventional local treatments, with marked improvement after oral finasteride and prednisone for the control of FFA, suggesting a beneficial action of the corticosteroid in these patients. Despite the positive result achieved in this case, more studies are needed to evaluate the role of systemic corticosteroids in patients with LPP and FFA.]

\section{REFERENCES}

1. Rao R, Sarda A, Khanna R, Balachandran C. Coexistence of frontal fibrosing alopecia with lichen planus pigmentosus. Int J Dermatol. 2014;53:622-4.

2. Kanwar AJ, Dogra S, Handa S, Parsad D, Radotra BD. A study of 124 Indian patients with lichen planus pigmentosus. Clin Exp Dermatol. 2003;28:481-5.

3. Dlova NC. Frontal fibrosing alopecia and lichen planus pigmentosus: Is there a link? Br J Dermatol. 2013;168:439-42.

4. Berliner JG, McCalmont TH, Price VH, Berger TG. Frontal fibrosing alopecia and lichen planus pigmentosus. J Am Acad Dermatol. 2014;71:e26-7.

5. Contin LA, Costa DM, Bet DL, Moura LB, Michalany A0, Di Chiacchio N. Líquen plano pigmentoso: tratamento cirúrgico com dermoabrasão. Surg Cosmet Dermatol 2014:6:384 --6.

6. James WD, Berger TG, Elston DM. Andrews - Doenças da pele. 10. ed. Rio de Janeiro: Elsevier; 2006. p.747-67.

7. Al-Mutairi N, El-Khalawany M. Clinicopathological characteristics of lichen planus pigmentosus and its response to tacrolimus ointment: An open label, nonrandomized, prospective study. J Eur Acad Dermatol Venereol. 2010;24:535-40.

8. Das A, Mishra V, Podder I, Kumar P, Das D, Das NK. Linear lichen planus pigmentosus: A rare entity with an illusory presentation. Pigment Int. 2014;1:100-2.
9. Duque Estrada B, Tamler C, Sodré CT, Barcaui CB, Pereira FBC. Padrão dermatoscópico das alopecias cicatriciais causadas por lúpus eritematoso discoide e líquen plano pilar. An Bras Dermatol. 2010;85:179-83.

10. Mulinari-Brenner F, Rosas FM, Sato MS, Werner B. Alopecia frontal fibrosante: relato de seis casos. An Bras Dermatol. 2007;82:439-44

\section{CORRESPONDENCE ADDRESS: \\ Fabiane Andrade Mulinari-Brenner \\ R. General Carneiro, 181 \\ Alto da Glória \\ 80060-900 Curitiba, PR - Brazil \\ E-mail:fmbrenner@ufpr.br}

How to cite this article: Mulinari-Brenner FA, Guilherme MR, Peretti MC, Werner B. Frontal fibrosing alopecia and lichen planus pigmentosus: diagnosis and therapeutic challenge. An Bras Dermatol. 2017;92(5 Suppl 1):79-81. 